\title{
THE NATURE AND TREATMENT OF STRABISMUS.
}

\author{
Dr. Citarles Delogé, \\ NICE, FRANCE.
}

Starting from Parinaud's conception of the nature of strabismus and the facts brought out by use of the diploscope the writer traces the double origin (nervous and optical) of strabismus. Educational treatment is indispensable, but must of ten succeed surgical intervention. The importance of the exhaustive study of each case is insisted on.

Oculists do not seem likely to agree among themselves on the nature and treatment of strabismus, despite the numerous treatises, some of quite recent date, which have appeared. The most varying opinions have been held and still find their convinced and authoritative defenders. The affection was, for some time, believed to be of muscular origin, thereby confounding it with deviation or squinting, which is an important symptom, but neither characteristic or always noticeable. Donders evolved really scientific explanations, and tho their value must not be exaggerated, it is certain that they are one of the greatest steps towards progress which has been accomplished. Javal, in turn, devoted a highly interesting volume of minute observations and documents to the theme.

Parinaud finally enlarged on the conceptions of his predecessors, and decided that strabismus was a faulty development in the apparatus of binocular sight. His opinion has become classic, and his masterly ideas have been exploited in the interesting work of Sauvineau. The given theory of a complaint has not alone an historical interest. It is generally admitted that any belief, be it true or false, gives rise almost always to appropriate actions. That is the case with strabismus, the treatment of which, after an entirely medical period, has become almost exclusively surgical; it has now entered a medicosurgical phase.

The subject has been treated so fully and repeatedly by so many eminent men that it may be pretentious in a newcomer to risk a few criticisms. Our excuse is, that in coming after them, we have profited by the ingenious apparatus our illustrious predecessors lacked. The ideas which we in turn, express are the result of numerous ob- servations made either in hospital or among our patients. We have been helped in the long and difficult work by the valuable counsel of Professor Lapersonne, our tutor, and the frequently recalled advice of Dr. Rémy, the inventor of the diploscope.

Dr. Rémy ${ }^{1}$ made his first experiments in the year 1901 ; since then some modifications of detail have been added to the diploscope, by several oculists and by himself; but these modifications have not altered the first conception. and the ingenious apparatus remains practically the same. The diploscope essentially consists in a large tube, closed behind by a plate moving on its own axis. This plate is pierced by four separate holes, one at 4 , the others at 6 centimeters. Two openings only are visible at the same time, owing to the action of the operculum. The apparatus is placed horizontally on a stem of $1.25 \mathrm{~m}$., ending at one end with a chin support, or two eye holes, and at the other with printed letters, of which the number varies, according to the nature of the experiment, between 2,3 or 4 . The rotation of the plate allows the numbers to be seen horizontally or vertically. Dr. Rémy has also constructed a smaller diploscope, differing from the preceding model, by its lesser length and the absence of the tube. The following illustrations will, we think, facilitate the comprehension of the simple technic of the apparatus.

The slightest alterations in binocular sight are immediately recognized by the displacement of the letters, which no longer appear in their normal place. but in crossed diplopia if there be divergent strabismus, or in homonymous diplopia, if the strabismus be convergent.

We shall not dwell upon the great developments of Parinaud's' theory, 
only we must insist on the fact that according to this author, strabismus is always an affection of childhood. In any case, his definition is extremely explicit. It is a question of "a flaw in the development of the apparatus of binocular sight, preventing the convergence of the eyes upon a fixed object." It is a disorder of central control in the reflex of the convergence. If this last be exaggerated, there is

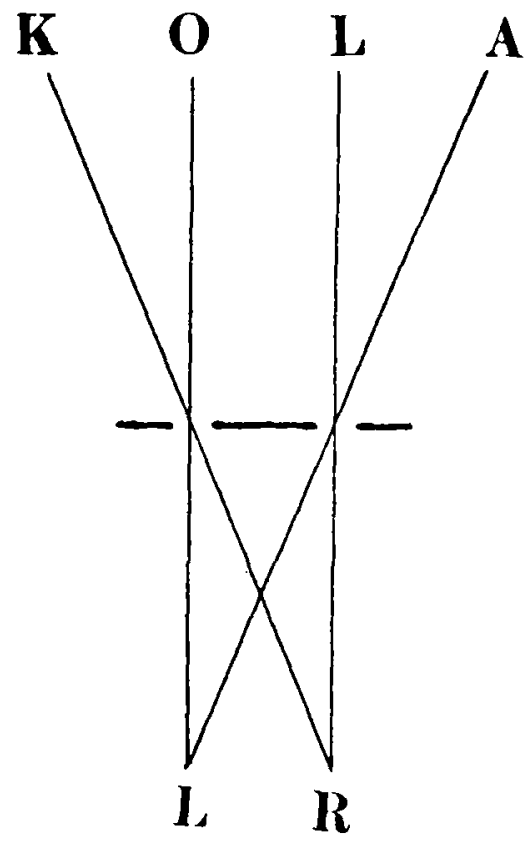

Fig. 1.-The iour-letter experiment for normal eyes. $R$. and $L$. are seen by the right eye. $O$. and $A$. by the left eye. The two openings furthest apart are placed horizontally for this experiment.

convergent strabismus; if it be lessened, divergent strabismus supervenes. The cerebral trouble itself may be primitive, and may, by that fact, prevent binocular sight, or it may supervene early and impede normal development. (Parinaud, pp. 19, 48, 50, $98,115,124$.) M. Sauvineau, ${ }^{3}$ in adopting this idea, wishes to localize the cerebral trouble more precisely still, to the center of the convergence (Sauvineau, loc. cit., p. 146).

Thus strabismus, which was according to savants a local affection of visual or muscular origin, became, according to Parinaud, an affection of the central control. The superiority of this conception over its often inadequate precedents cannot be ignored. It appears to be not only the most natural but the only probable explanation of certain forms of strabismus, more particularly those which supervene when optical causes are at fault. Two children possess the same refraction, the same visual acuteness, one has a direct gaze and normal binocular sight, while the other is strabismic. There

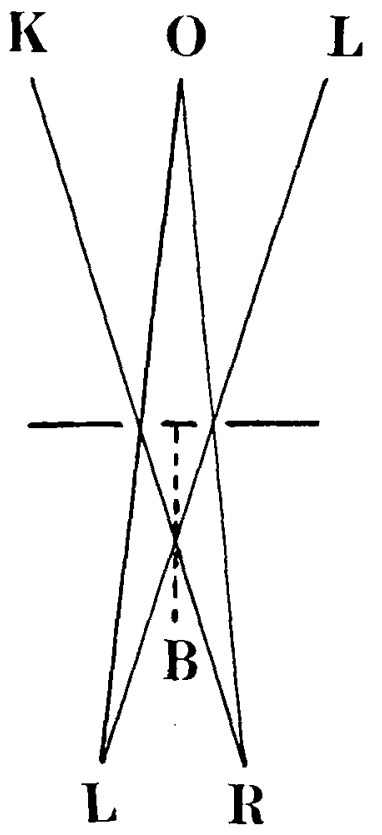

Fig. 2,-Three-letter experiment for normal eyes. $K$. and $O$. are scen by the right eye. $O$. and $I$., are seen by the left eye. If the bar placed ver tically in front of the tube is Iowered $O$ alone contically in front of the tube is lowered, $O$. alone continues to be seen by both eyes, $K$. and 1 . hav ing their rays intercepted. The two nearer open-

must be a cause for the latter condition, and since it does not exist in the eyes, it must be sought elsewhere, that is, in the brain. Strabismus is, so to speak, the sign of organic decadence, a stigmata of degeneration.

The important part played by all sorts of infectious maladies, measles, scarlatina, typhoid fever, in the case of the child, is thus wonderfully explained. Convulsions, it is well known, are frequent in the previous life of the little strabismic. Professor Fournier and Dr. Antonelli have, on their side, pointed out, perhaps rather immoder- 
ately, the importance of syphilis in the progenitors; also, there is the question of consanguineous marriage, or of alcoholism. To sum up, it secmed probable that all causes capable of bringing nervous troubles, could in a certain measure disorder the delicate machinery of binocular sight.

The nervous theory of Parinaud would, if necessary, serve a clinical argument. Strabismic children are often not only irritable and nervous subjects and actual neuropaths, but strabismus itself presents at times considerable individual differences. All those who have treated children know that tho strabismus may rapidly improve under the infuence of glasses and atropin, there are unhappily fairly numerous cases where the best informed usual therapeutics show themselves particularly inefficacious, whether they be applied to the generally convergent form of the strabismus itself, or to the preexistent nervous cause.

But if Parinaud's theory throws a new light on cases of strabismus that supervene where the child's eyesight is almost normal, it becomes less necessary, tho still very useful, in explaining the form of strabismus that Donders has attached to refraction troubles, and principally to the discord they establish between accommodation and convergence. In fact, the greater part of these strabismics generally give way fairly quickly to suitable optical treatment. Consequently, it becomes difficult to determine the part of ametropia and that of the nervous system, in the evolution of the ocular affection. The interpretation may become singularly embarrassing, and a minute examination of the subject and its antecedents will be found necessary to specify the etiology.

Regarding strabismus in the adult, Drs. Parinaud and Sauvineau are most discreet. If they speak of it, they call it an old strabismus, or explain it by a nervous anterior predisposition. Our intention is to show precisely that this affection can supervene among individuals whose binocular sight is perfectly developed, and without necessarily being connected with special nervous in- fluences. This variety of strabismus is a particularly interesting study, because it is peculiar to adults, and its evolution can, so to speak, be easily followed from day to day. But, before we go into the numerous points which form the basis of this study, it would not be uninteresting to come to terms with strabismus itself.

Strabismus must not be identified with deviation, which may not be present in strabismus, but may appear in totally different disorders such as paralysis, or tumors of the orbit. Comitant strabismus is essentially a disease of binocular sight, preventing the convergence of the eyes upon a fixed object. The visual trouble varies according to the evolution of the strabismus.

Normal binocular sight is first attacked by simultaneous vision, in which both eyes, tho one is often weakened, preserve their visual powers. Thence the patient experiences more or less marked inconvenience, rather indefinite and generally of short duration, during which deviation is rarely apparent.

This period is soon succeeded by partial simultaneous sight (simultaneous sight of Parinaud) which we have elsewhere called panoramic vision. Parinaud has defined it very well in further naming it alternating central vision. Neutralization (suppression, inhibition) is the most typical and curious characteristic of this period. However, as neutralization has its adversaries, it is necessary to agree upon it. Parinaud, in his treatise, says of it, "The physiologist looks upon it as a word without significance," (p. 96) and since then the criticism, given in superb style, has enjoyed extraordinary popularity. Indced, one must not be led away by words, since, whatever the cause, neutralization is distinctly one of the most clearly proved facts, not only with regard to sight, but to the other senses. The more the latter become complex, the more they teach us, and the more they neutralize. As a principal effect, the attention we bring to bear on a subject lifts it from its frame and separates it from surrounding con- 
tingencies. In a word neutralization is not a particular act of the sight, but a general phenomenon, as necessary to our intellectual activity as the shades are to the lights of a picture. This is so true that in some nervous pathologic conditions fatigue will be enough to lessen this faculty and bring about different and trying disturbances. We ourselves have come across many strabismic patients who suffered almost continually from diplopia.

In panoramic sight, neutralization is the reaction of the defense against diplopia, and the more rapidly it intervenes, the less the subject suffers from the preceding period. 'This neutralization is elective and kecps exclusively to that portion of the retina corresponding to the object seen by the nondeviating eye, and avoiding the other reglons of the visual field. For this reason we have called this form of sight panoramic. The diploscope makes the study of neutralization an easy matter, and by the judicious use of this instrument, it can often be made to appear and disappear at will. At any rate, alternate is the opposite of simultaneous and nothing is more certain than alternating central sight, if it be not alternating central neutralization. There has been a mere wordy quarrel concerning it, and we hold, nevertheless, to the neutralization admitted by Graefe and Javal as a characteristic and indisputable fact.

Neutralization does not always conduct itself along the same lines. It is alternating and generally remains so. when both eyes are of equal strength; or when one is far-sighted and the other near-sighted.

But at times, without appreciable cause, or with more reason, where one of the two eyes is manifestly inferior to the other, neutralization becomes fixed; and alternatively the sight becomes monocular. This new stage is the graver on account of the momentous disorders it brings about. One of the most embarrassing of these accidents is amblyopia ex anopsia, so called because it is not accompanied by any appreciable alteration, sufficient to explain it. It is frequent and often considerable in the case of a child attacked by convergent strabismus. It is much less marked in cases of myopia, or, when an adult is attacked by divergent myopic strabismus. Even when of long duration, the functional diminution of sight rarely exceeds a few tenths. An exact optical correction and a few exercises will, in this case, generally suffice to bring about a rapid and complete restoration of the sight.

Simultaneous vision, alternating central or panoramic vision, monocular sight, these are the stepping stones, the successive and characteristic stages of strabismus. In the course of this affection, amblyopia and deviation itself, are but frequent and simple, tho often very annoying accidents.

Strabismus, thus understood, may make is appearance in an adult endowed with a perfectly developed apparatus of binocular sight. This certainty, with us, is the result of numerous minute and most affirmative observations. If the contrary idea has been overvalued or overpraised, it is certainly due to the difficulty of examination, and the imperfection of the apparatus employed. Rémy's diploscopes have allowed us to follow the evolution of strabismus, in a large number of patients of varying ages. For the sake of precision, we will give a summary of some of these cases further on.

An appreciable difference in the sight of the eyes is the usual cause of strabismus in the adult. Hering compares the optical apparatus to "a double team driven by single reins." Ve will use his ingenious idea. If one of the team goes lame, it either stumbles on with difficulty or stops and must be detached. It is the same case with the sight. The causes of strabismus in the adult, are ther: very varied. A unilateral and rather tardy myopia. a traumatic cataract, monocular aphakia and, lastly, to generalize, any accident which brings about an appreciable difference in the sight of the eyes, enter into the question. We have recounted thirty cases of anisometropia, chosen from many others, in our inaugural treatise. Fach time the difference of refraction exceeded two di- 
opters, a visual alteration, an actual strabismus existed before correction. Dr. Sauvineau, who cites this fact, adds however, further on, that he has not much belief in the influence of anisometropia as an essential factor of strabismus. We reply to this that if among the patients examined, a certain number were attacked by anisometropia in childhood, others were numerous, who did not suffer until adult age; when, so to speak, the developnent of the apparatus of binocular sight was perfect.

Thus, strabismus is infinitely more frequent than is generally believed, and a methodical examination, with the diploscope, of persons afflicted with defective sight gives adequate conviction. The numerous researches we have made, since our treatise, confirm this vicw.

It would be no doubt exaggeration to say strabismus presents special characteristics, which distinguish it from previously described forms. Tho lacking in very marked differences, strabismus is, howcver, accompanied by slightly peculiar symptoms. The onset of strabismus in the adult as in the child, is slow and progressive, and of insidious growth. Also, apart from the more or less acute sight troubles, usually attributed to fatigue, the patient rarely complains. Deviation is more interesting. At times, it is very marked, but more often it remains trifling and it is chiefly in such cases that invisible strabismus (heterophoria) is frequent. On interrogation, however one often learns that a certain amount of deviation in the vague gaze, has sometimes been noticed. Stereoscopic vision may exist and the diploscope is almost indispensable to make the alterations in binocular vision manifest. Let us also remark that large as well as small diploscopes are necessary, for the sight may act differently near to, or at a distance. Deviation, apparent or not, is nearly always produced from outside, and divergent strabismus is much more frequent.

Amplyopia ex anopsia is less pronounced. It has surprised us more than once to find patients, who had suffered from unilateral myopia for twenty years and who, even in reading use their emmetropic eye, obtain almost immediately, excellent sight with their correction. In short, this strabismus is more frequently seen among perfectly balanced and healthy indivicluals, with whom the affection is ocular, and purely accidental.

$\mathrm{We}$ will point out later, in speaking of treatment, the very great curability of this form of strabismus. We will now cite, as bricfly as possible, the following cases:

CASE I. The patient was a boy of 14 years of age, whose left eye had become myopic following keratitis. We saw him for the first time at the Hotel-Dicu, on the 15th of May, 1905. According to the skiascope, M. Me. . . is emmetropic in the right eye and myopic in the left $4.50 \mathrm{D}$.

$V$ ision R. $=0.9$. In $\mathrm{L} .=0.1$, after correction 0.9 .

We examined him diploscopically. His sight, without correction is alternative, and his myopic eye serves for nearer sight. After correction, neutralization persisted for a time, then simultaneous vision appeared, acting divergently. Binocular sight commenced to become normal at moments, two days afterwards, following a course of exercises. By the 20th of May, the patient had acquired normal binocular sight, without effort. He wore his correction. His sight was still good, when we saw him again, three months later.

This patient is of interest for two reasons. No deviation was apparent, and stercoscopic vision came on directly after correction. Moreover, this patient also had strabismic sight.

CASE II. The case of Mlle. Em. Mart, aged 17 , is rather different. We saw this patient on the 2 nd of August, 1904. at the Hotel-Dieu. She presented a paracentral leucoma of the right eye and myopic astigmatism.

Skiascopy: R.: Vertical-11. D., horizontal-6. D.

Ophthalmometer: R. = 5. D. Ast. I. = Normal.

The sight of the right eye after correction, equalled 0.5 only. This girl squinted very markedly outwards and 
a little above, but this deviation was not visible at first. With the large diploscope it took a half hour of rather trying exercises to make the neutralization, which was alternating, disappear. Her sight soon became simultaneous, and the diploscope revealed a divergent and sursumvergent strabismus, of which we already felt sure.

Next day, her sight was still simultaneous, and the letters seen by the right eye, armed with its correction, appeared much smaller. We diminished the strength of the spherical glass by $1.50 \mathrm{D}$. and immediately the letters became equal. Two days late $r$ we returned to our first correction: R. E. (horizontal axis $-5 .-6$.) and the letters remained the same size. The sight of the right eye is now 0.9 . The binocular vision is normal. In this case, exercises for six days sufficed to cure a strong ametropia, complicated by astigmatism, and a double deviation, one of which was quite considerable. Scen two months later, the sight of Miss Mart . . remained perfect.

C...E III. Here is a quite personal experience. "We have systematically corrected one of our eyes, for some days, tho both are almost equally myopic. 5 dioptres. Some days we have experienced a rather violent disturbanct. ind evident ocular fatigue. The chat se of sight, tho very appreciable, was rather difficult to define. To the unc $\varepsilon_{i}$, rected eye, the image was naturally much less clear and of diffused outlines. I iterally speaking, no diplopia existed, but rather a superposition of objects, unequal in drawing and size. Iight chicfly showed this disturbance. Seen in front and at a certain distance, the gas jet had the following appearance: The jet and its cylinder of very precise and very luminous contour, were in the center, surrounded by a very widcly diffused object, without clear limits. As soon as the vision became slightly lateral, the two reflections persisted, but without preserving their relation, and the more the vision became eccentric, the farther away they drew from each other. After a week, the habit was acquired and a strong tendency to deviation in the left eye was remarked at this mument; the vision was clear. 'The trouble persisted rather longer with very luminous objects. The integral correction of our eyes was speedily followed by a return of binocular vision, still, it required some days to become perfect and natural.

We have dwelt upon our case because it is very significant. A difference of 5 diopters very rapidly determines simultaneous vision, which corresponds to the trouble experienced, then neutralization intervenes, the sight becomes clear, but only one eye acts, and the deviation of the left eye becomes evident.

Other Observations. - W We do not wish to extend the detail of these observations. We will, however, quote the case of Dr. G- of Paris. This colleague, who was about thirty-five years of age, had, for a long time, an unilateral myopia of 6 diopters and $y$ divergent strabismus of 32 degrees. The sight of this eye was normal, after correction, the convergence being very well preserved. It was decided to operate. Nevertheless, on our insistence, his physician allowed us three weeks in which to try orthoptic treatment. Neutralization was difficult to conquer; and to revive diplopia we had to employ 70 diopters of prisms, afterwards rapidly lessened. After six treatments, which did not, on an average, last longer than thrce quarters of an hour, the sight became normal. Afterwards, nur colleague, provided with his correction, found no difficulty in driving his automobile.

To this case may be added the following observations of Parinaud:

$A$ young man of 14 suffering from convergent strabisnus of the right eye, of 30 degrees, had myopia of 8 diopters and a hypermetropia of $0.5 \mathrm{D}$. in the left. "After a year, the strabismus completely disappeared under anisomctropic correction."

Tho recognizing that the treatment, logical as it was, had been employed in a totally empirical manner. this cure certainly goes to confirm our ideas on the origin and treatment of certain strabisms.

Lastly, if trouble is taken to seriously examine the eyes of patients suf- 
fering from monocular aphakia, which case is of fairly frequent occurrence, in hospitals, a true strabismus, which will only yield to suitable exercises will be invariably remarked, before as well as after correction.

These few examples could be largely multiplied from the number that exist. It seems that the conclusion drawn from them insists on a place for strabismus of a purely visual origin, besides strabismus whose primitive nervous origin appears indisputable. And this gives justice to those eminent scientists like Javal, who have defended the optical origin of strabismus. Their arguments were true and they were often in the right. This remark does not tend to lessen Parinaud's merit, for it is very difficult, nay, even impossible, to cover all cases of strabismus with a single formula. If the well known French axiom be true, "Il n'y a pas de maladies, mais des malades" (there are not diseases but patients), it has been written of strabismus, for an affection with more varying symptoms does not perhaps exist. It may be said without exaggeration that one does not come across two strabismics in a hundred exactly alike, so much does the individual character show itself in the smallest details. In any case, theories are but relatively truthful, however ingenious they may be. They are imagined to explain and arrange facts, and ought to be mixed with a slight indifference, an indiscreet curiosity.

Otherwise the dangerous custom of giving facts an erroneous interpretation is acquired. We have undertaken

- this work because it seems to us that a too important part in the treatment of strabismus is given to overtheoretic ideas, estimating that it would be expedient, in various clinical affections, to oppose, within limits, an appropriate therapeutics.

The treatment of strabismus is at present understood in very different ways by the greater number of oculists, with no precise rule in its application, and many variations of detail. In any case modifications only deal with operative treatment, which alone counts with the majority of practitioners.
It seem to us that the time has come to apply a little more method and precision to the treatment of so common an ailment. That it is a particularly delicate and embarrassing work is not to be denied. It is not, however, a question of employing any new method, only of utilizing, with more advantage, relatively old aids, and of conciliating as much as possible therapeutics and clinical instruction.

Convergent strabismus is particularly frequent in children, but its origin is not always recognized as the same. It is very often allied to a neivous central disorder (Parinaud), at times the influence of refraction is shown (Donders); and in other cases these two causes mingle so closely that it is impossible to say which part is due to the nervous system, and for which abnormal refraction is responsible. Therefore, we will not attempt to lay down a treatment for these etiologic varieties. In fact, such precision seems to us impossible, and the practitioner should above all be inspired by circumstances.

The greater number of authors agree on the treatment of convergent strabismus, and differences of opition are chiefly addressed to questions of detail. In one of his lectures published by the Presse Médicale, Professor de Lapersonne has magisterially studie.! operative indications in strabismus. $\mathrm{He}$ has clearly and precisely pointed oi the precepts to be drawn from age, efraction, visual acuteness, from th ${ }^{\wedge}$ degree of strabismus, and from the paitent's antecedents. We cannot do better than to repeat them to our readers.

Speaking of surgical treatment, he insists on the reason for rejecting too hasty intervention, that is, before the patient is ten years old. Too early operation may result in complete failure, and even in a disaster difficult to repair. We will not speak of operations for strabismus; their indications and technic have been set forth with precise and circumstantial detail in Dr. Terrien's fine "Treatise on Ocular Surgery."

When a child suffers from strabismus the primary treatment consists in an earnest and serious examination, 
and an exact optical correction. Orthoptic treatment ought, afterwards, to be seriously prescribed, whenever visual acuteness is normal, or tends to become adequate. We here give some rather important details on this subject.

The strabismic child very often presents amblyopia ex anopsia of one of its eyes. This amblyopia is considerable at times, and prevents all reeducation of binocular sight; therefore, our efforts should, first of all, ,be centered on its cure. The greater number of specialists order as an efficacious means, the wearing of an occlusive band, or an opaque disc, on the healthy eye. This, according to our idea, is a means that should never be employed, unless one is forced to it: it has the serious disadvantage of being long, tiresome, and only too often inefficacious. Amblyopia submits, with bad grace, to this treatment; and on the other hand, it is satisfied with rather weak visual acuteness, which it does not try to further enlarge.

As we have already said elsewhere, it is better to make the patient exercise under supervision. After bandaging the healthy eye, the letters, which assist visual acuteness, are brought rather close to the patient, so that he can distinctly read the largest characters with his amblyopic eye; they are then progressively drawn away. We have, in some cases, been able to increase the visual acuteness of the amblyopic eye by several tenths with a single treatment. In spite of all efforts, in other cases the acuteness makes very slow progress. The only fault of this treatment is the fatigue it imposes on youthful patients, which necessitates, particularly at first, exercises of very short duration.

As soon as visual acuteness attains at least a tenth, orthoptic exercises ought to commence. Javal had recourse to the stereoscope, and it is on this instrument, more or less modified. that most oculists depend. Convergent strabismus can certainly be cured in this way, only the use of the stereoscope in such an affection appears almost paradoxical. Indeed stereoscopic sight demands parallel ocular axes, that is to say, of relative divergence. Doubtless this fact explains the very great difficulty which this treatment presents. Moreover, the stereoscope has other faults no less grave. Experiments with this apparatus are difficult of control, exercises can only be made at a short distance, and above all, stereoscopic vision differs from binocular vision. It seems, then, once divergent strabismus cured, that this apparatus may be of use with a view to obtaining slight surcorrection. Its use after the cure of convergent strabismus would not be advisable.

The length and difficulty of such a method had doubtless far from encouraging results. The following phrase of Javal, quoted by Professor de Iapersonne, demonstrates this fact: "The reestablishment of binocular vision by exercises usually requires as long a time as that which has elapsed since the commencement of deviation. A child of eight who commenced to squint at four may be cured at twelve years of aye."

Many other forms of apparatus have been enployed since this epoch, such as the amblyoscope. But among them all, Rémy's diploscope appears to us to assure the best and easiest results. The marvelous precision of this instrument, its variety of exercises and the ease with which it is understood and may be controlled at every instant, allow of its use, even with very young children. Whe have treated a four-yearold baby, by replacing the letters, which he did not know, by the figures of animals. His German governess explained the movements to him and we have obtained the most excellent and unhoped for results.

The end to be attained in the first place is diplopia. With a little practice in varying the letters, and the intensity of light... neutralization is generally vanquished fairly quickly. Consequently, the exercises assist two objects; they make the amblyopic eye work, and strengthen its acuteness; they contribute at the same time to lessen the deviation of the cyes.

If taken in good time, and if secondary modifications have not already appearcd in the muscular apparatus, 
there is every chance of obtaining entire success. It must not, however, be denied that the cure of strabismus by exercises is, as a rule, long and arduous. It demands the hearty cooperation of the patient, and unending patience in parents and doctor. There are many reasons for this, but the greatest of all is the difficulty of obtaining divergent movements of certain duration. Still, as good results follow fairly frequently, one must not despair too quickly.

Even where these means fail to cure, the exercises prepare the ground in a wonderful manner for surgical intervention. To commence with, they cure the amblyopia ex anopsia, they revive diplopia and finally and notably reduce deviation. The surgeon will know exactly what the operation demands and it will be performed under the best possible conditions. Even then, the oculist's part is not ended. In an immense majority of cases, no operation, however successful, gives more than a cosmetic result. It is therefore the moment to continue orthoptic exercises, which are alone capable of assuring a complete and definite result, by inducing perfect binocular vision.

While on this subject, we cannot agree with the many practitioners who content themselves with advising parents to buy a stereoscope, and some Javal's cards. Stereoscopic exercises, interesting tho they be, are too difficult to control. The doctor's presence is a necessity, otherwise parents and patient quickly relinquish the instrument. Diploscopic treatment is preferable, on account of its rapidity, as well as for reasons already given. Stereoscopic vision should not be exercised until the patient can easily do the exercises of both diploscopes, and then only with a view to obtaining slight overcorrection. Naturally optical correction must not be neglected, whenever it may be useful to increase visual acuteness.

Thus, the cure of convergent strabismus is far from being the simple and easy matter it is so wrongly thought to be. On the contrary, it demands sustained effort from the patient and necessitates in the doctor the closest vigilance and much facility in the practice of exercises.

\section{DIVERGENT STRABISMUS.}

Even in the most justly appreciated and widely spread treatises, the accepted opinions on divergent strabismus seem to us to call for a few reservations. With the exception of the most recent works, the greater part of which have appeared in periodicals, their authors are, more or less, partisans of surgical intervention. With them, operation is the chief element of success, and orthoptic exercises are of much less importance, having only a complementary value.

This idea, shared, moreover, by the majority of oculists, depends on various reasonings. The reports on convergence and the much narrower accommodation here, the slow evolution which often precedes insufficient convergence and its habitual tendency to increase, help to render divergent strabismus rebellious to all optical treatment. Thus, operation was advised in the greater part of strabismic cases, even when the strabismus was unimportant and periodic.

We have not agreed with this opinion for a long time. In all that concerns divergent strabismus, we repeat what we have said with regard to convergent strabismus; each affection is distinct and requires careful study, not only of its actual symptoms, but as far as possible, of its probable causes.

Indeed, it is very certain that in this form of strabismus the reports of convergence and accommodation are far from bringing about such direct consequences, and it is equally certain that evolution here tends towards aggravation rather than cure. Does this mean that we must look upon dioptric treatment as useliess and relegate it to the position of a mere accessory? We do not believe so and we will explain why.

To do so, we must insist upon two essential points intimately allied to the pathogeny and treatment of divergent strabismus. Divergent strabismus and insufficient convergence have been united to such an extent by so many authorities that it is difficult to take the other side. In fact, strabismus, ac- 
cording to them, is very often preceded by more or less congenital insufficiency of convergence. There is some truth and considerable error in this view.

It often happens, and Parinaud, with perfect right, insists on the fact, that insufficient convergence is the first distant cause of divergent strabismus, which appears at a very much later date. This insufficiency appears to be of nervous origin, and it is, moreover, seldom accompanied by refractive troubles. The sight of each eye is normal. As soon as the patient makes efforts at convergence, one of the eyes becomes fixed and does not pass over the median line. Fatigue and even pain quickly follow a prolonged effort, without a sensible increase of convergence. In the adult, this insufficiency frequently develops into a more or less important strabismus. But such cases secm rather rare; at least, they have always been exceptional in the cases of strabismus we have attended.

Besides this ordinary insufficiency, which is very prominent and generally congenital, one might almost say essential, there exists another insufficiency of convergence, which often attacks both eyes, but as a consequence of strabismus instead of a cause. The absence of convergent movements brings about fatigue, and actual muscular impotence. It is found in the careful examination of patients suffering from a fairly old divergent strabismus, that sustained efforts at convergence very rapidly produce in the ocular globes, badly coordinated oscillatory movements, which tend to a very imperfect convergence. It is therefone a question of acquired weakness, not functional impossibility, for if patients are submitted to continuous appropriate exercises the rapid reestablishment of functional convergence is nearly always brought about. On the other hand, the insufficiency may be due to other motives, and it may, in certain cases, be entirely at fault. We can recall patients who suffered for ten years and more from divergent strabismus, without presenting the very slightest degree of insufficient convergence.

Another and no less important point of which we have already spoken con- cerns the optical origin of divergent strabismus. The most common cause of adult strabismus, outside those injuries and disorders of the eye capable of compromising binocular vision seriously enough to authorize its reestablishment, is anisometropia, that is to say, a difference in refraction between the two eyes. For some time the trouble induced is perhaps more real than apparent; the ocular movements seem normal, the convergence itself appears unaltered. Nevertheless a closer examination demonstrates the almost complete destruction of binocular sight, and we learn on interrogation that the gaze deviates at times. This deviation often becomes more apparent than pronounced, at other times it develops into an evident and even important divergent strabismus.

It is necessary to lay stress on these two factors, so important is their part in therapeutics. We will now demonstrate that they really constitute the greatest obstacles to a cure.

Many authors agree in asserting the impotence of dioptric treatment in divergent strabismus. This impotence is, in great part, due to the inefficacious remedies opposed to anisometropia and insufficient convergence.

Let us put the strabismus united to congenital convergence on one side. It is rather a case, and is less a question of treating strabismus than of remedying insufficiency. If medical treatment appears incapable of success, there is always the resource of surgical intervention, after which a few exercises generally suffice to restore binocular vision.

Divergent strabismus is frequently united to anisometropia. It must be acknowledged that an entirely empirical treatment has been too long looked upon as a cure. The patient, once provided with an excellent correction, should, without further indications, accustom himself to this correction. Ignorance as to the disorders of binocular vision, and the absence of controlled apparatus were responsible for this way of thinking. Clinical surprises are often curious, and it has occurred that some patients have been more or less rapidly cured by this means. Generally, 
they have quickly given up the correction, which, far from ameliorating the sight, became a sources of serious inconveniences, such as vertigo, nervous headache, false relief, and dizziness. Taught by these facts, practitioners have wisely dissuaded the correction of anisometropia greater than a diopter and a half.

In our turn, aided by Rémy's diploscope, we have resumed this study, and found reasons to oppose an integral correction. These reasons are two in number; strabismic deviation - the cause of more or less obvious diplopia, which is often sufficient to awaken improved visual acuteness-and the unequal size of objects seen by each eye. Many years of experience convince us that the inequality in the size of objects, which is such a trouble to the patient in the beginning, never persists. It is not so with deviation; this requires a more or less lengthy treatment, and only yields to a course of suitable exercises.

We lay stress on the fact that the difficulty in curing an anisometropia and its accompanying deviation does not depend on a more or less marked dioptric difference-an anisometropia of ten diopters may be much easier to cure than another of three diopters. It is equally independent of the greatness of the deviation itself. The real difficulty arises from the insufficient degree of convergence. That it is which causes all delays in cure, therefore our first effort ought to be to diminish and vanquish it. Convergence once established, victory is at hand, for the reeducation of binocular vision becomes henceforward rapid and easy.

The habitual treatment of insufficient convergence ought always to be pleasing. If it is a question of a slight degree, the wearing of prisms, or the decentering of corrective glasses is generally advised. This method presents some grave disadvantages; it makes an inadequate, heavy, and sometimes dangerous palliative.

The insufficient convergence common to strabismus is far from having absolute stability, therefore prismatic correction has not all the precision one could wish. On the other hand, if it exceeds, by ever so little, a few diopters, the glasses become too thick. One may even add that the cases which might be benefited by this treatment are rare. Lastly, and herein lies its principal fault, this method does away with all serious and useful efforts on the part of the patient. Now, it would be better, if possible, to reestablish an important function, all the more easily cured, because frequently united to a weakness due to actual alteration. For that reason, the procedure of reeducating convergence is more preferable. Tho undoubtedly more difficult, it has the incontestable advantage of curing the affection and may be applied to all sorts of cases, even to those of a degree opposed to prism correction.

Stereoscopic exercises are often prescribed to improve insufficiency. This method, faulty as it is,--we have given the reasons,--may be of service. But it must never be more than a preliminary treatment for strabismus endowed with stereoscopic vision. Completion of cure demands diploscopic exercises.

When insufficiency is accompanied by marked deviation the greater number of oculists indicate operation as the best treatment. We have given our opinion on surgical intervention in such cases. In itself incapable of producing a cure, it gives a mere cosmetic result, and ought to be invariably followed by reeducatory exercises. Nor do we believe that the duration of this indispensable work is sensibly diminished by operation, for we have seen important divergent strabisms cured in less than ten treatments by the orthoptic method alone. Outside those cases where reeducation is impossible, one must never, as is often so mistakenly advised, operate at the first onset. A wisely conducted medical treatment will usually suffice, and the patient will gratefully avoid intervention that can, however, in case of necessity, be resorted to later.

We will rapidly sum up our usual course of treatment in the greater part of cases of divergent strabismus.

In the first place integral correction of the ametropia, no matter what the 
degree, or how marked the difference in refraction between the two eyes.

If this correction is not sufficient to stop neutralization, and this happens frequently where the deviation is very marked, we try to revive diplopia. Many excellent means may be utilized to this end either simultaneously or separately. Diploscopic exercises, colored glasses, and different degrees of light will often suffice. In rebellious cases, prisms must be resorted to. We have employed 70 diopters of prisms, in the case of one of our patients (Dr. GOrdinary prisms may be used. Dr. Rémy's rules are most convenient, but the turning prisms of Landolt or Risley seem to us to be most favorable.

As to faults of insufficiency and convergence they ought to be opposed by exercises adapted to the type of the deviation, the enumeration of which would alone lead to unnecessary length. First experiences are often painful and trying (they brought on syncope in one case) but the patient quickly gets accustomed and interested in his progress, and given determination, the cure will be rapid and complete.

In conclusion, we wish to say a few words regarding two fairly frequent cases. Divergent strabismus frequently coexists with slight vertical deviation. Each time we have come across it, it has disappeared, with exercises, at the same time as the horizontal deviation.

The following observations concern certain pronounced squints due to considerable anisometropia. In an affection of this class ( 15 diopters of anisometropia and 22 degrees of divergent strabismus) we have been astonished that the patient, who had perfect binocular vision, with the diploscope, continued to see double in the street. We had therefore to proceed to the reeducation of peripheric binocular vision. We used Professor de Lapersonne's perimeter with perfect results. The use of this instrument is certainly more practical than the mural method prescribed by Lagrange.

Such is briefly recapitulated the treatment that at present seems to us the best means of curing divergent strabismus; it is decidedly the most rational. Without doubt it demands a certain apprenticeship in the beginning and may on that account seem too lengthy. But a little experience and custom will rapidly bring about the discovery of a more rapid process and short cuts that will save precious time.

CONCLUSION.

Owing to its double nervous and optical origin convergent strabismus requires a treatment that is long and particularly delicate to establish, but in which reeducation of binocular vision is essential. Where possible this constitutes the preferable method, as well as the indispensable complement to surgical intervention. We ought to beware of the opinion of a great number of excellent oculists, who believe divergent strabismus can only be relieved by surgery. With a few reserves, we hold the contrary to be the truth, and it is chiefly in this case that orthoptic treatment gives the most rapid results and brilliant successes.

The first conditions of treatment are an exhaustive study of each case and a profound knowledge of facts. In spite of which, the not alone apparent, but true cure of strabismus frequently remains a very real difficulty; but this fact, far from discouraging us, ought rather serve to render us more exacting in the end to be attained and more severe in our choice of the right means with which to obtain it.

\section{REFERENCES.}

1. Delogé. Arch. d'Opht. Dec., 1904.

2. Parinaud. Le Strabisme et son traitment. Paris, 1899. O. Doin.

3. Sauvineau. Bull. de l'Acad. de Med., v. 81, p. 789 .

4. Rémy. Recueil d'Opht., 1901, p. 385; 1902, p. 1, 70, 583; 1910, p. 53. 